\title{
Alterações das Propriedades Funcionais e Estruturais de Grandes Artérias no Diabetes Mellitus
}

\author{
revisão
}

\section{luiz Aparecido Bortolotto}

Unidade de Hipertensão, Instituto do Coração (InCor), São Paulo, SP.

Recebido em 18/17/06 Aceito em 23/17/06
RESUMO

Alterações funcionais e estruturais das grandes artérias exercem um importante papel na patogênese das doenças cardiovasculares. O diabetes mellitus, ao lado da hipertensão arterial e do envelhecimento, pode induzir essas alterações em diferentes territórios arteriais, e assim levar ao desenvolvimento de aterosclerose e suas conseqüências cardiovasculares. A principal alteração da função das grandes artérias é o aumento da rigidez, enquanto que a principal alteração estrutural é o maior espessamento da camada intima-media da artéria carótida, encontradas em ambos os tipos 1 e 2 de diabetes. Os mecanismos destas alterações estruturais e funcionais arteriais no diabetes incluem a resistência à insulina, o acúmulo de colágeno devido à glicação enzimática inadequada, disfunção endotelial e do sistema nervoso autônomo. $\mathrm{O}$ aumento de rigidez arterial é um marcador de risco cardiovascular em pacientes diabéticos, e o tratamento tanto do diabetes per se quanto de dislipidemia e hipertensão arterial associadas pode modificar beneficamente essas alterações arteriais. (Arq Bras Endocrinol Metab 2007;51/2:176-184)

Descritores: Rigidez arterial; Diabetes mellitus; Velocidade de onda de pulso; Espessura íntima-medial

\section{ABSTRACT}

Modifications of Structural and Functional Properties of Large Arteries in Diabetes Mellitus.

Functional and structural modifications in large arteries play an important role in the pathogenesis of cardiovascular diseases. The diabetes mellitus besides arterial hypertension and ageing can induce these alterations in different arterial sites, and so leading to the development of atherosclerosis and its cardiovascular consequences. The main functional change of large arteries is an increase of stiffness, while the main structural modification is an increase of the intima-media thickness of carotid artery, and both changes have been recognized in both type 1 and type 2 diabetes. The mechanisms of these structural and functional arterial modifications in diabetes include insulin resistance, collagen increase due to inadequate enzymatic glycation, endothelial and autonomic dysfunction. The increase of arterial stiffness is an independent cardiovascular risk marker in diabetic patients, and the treatment of diabetes per se and even of associated dyslipidemia and arterial hypertension can favorably modify these arterial changes. (Arq Bras Endocrinol Metab 2007;51/2:176-184)

Keywords: Arterial stiffness; Diabetes mellitus; Pulse wave velocity; Intima-media thickness 
$\mathrm{H}$ Á UM CONHECIMENTO CRESCENTE e recente de que a função anormal das grandes artérias exerce um papel importante na patogênese da doença cardiovascular. Determinações mais precisas das propriedades elásticas da aorta e das grandes artérias, incluindo medidas angiográficas (1), ecocardiografia (2), medidas por ultra-som da aorta abdominal e do diâmetro carotídeo, ressonância nuclear magnética (3), angiografia radioisotópica (4) e velocidade de onda de pulso (5), têm mostrado associações das alterações dessas propriedades com eventos cardiovasculares e também com aterosclerose coronária em diferentes populações. Dentre estes diferentes métodos, os mais utilizados em estudos populacionais são os que avaliam a rigidez arterial pela medida da velocidade de onda de pulso (VOP) e os que medem a estrutura e função da artéria carótida por método ultra-sonográfico.

A medida da VOP, sobretudo as do segmento carotídeo-femural, que reflete a distensibilidade da aorta, tem boa reprodutibilidade e fácil realização, principalmente em estudos envolvendo vários indivíduos. Um esquema do procedimento é apresentado na figura 1. Ao se medir a VOP carótida-femural, por exemplo, estamos obtendo um índice indireto da distensibilidade aórtica e, conseqüentemente, da sua rigidez. Assim, uma VOP muito elevada representa uma maior rigidez arterial no segmento estudado. Nós demonstramos em diferentes estudos que a VOP aórtica é um marcador de doença aterosclerótica (6) e também está associado a outros marcadores da doença como a homocisteína (7).

Diversos fatores de risco cardiovascular podem afetar as propriedades funcionais e estruturais das grandes artérias e conseqüentemente promover aumento da rigidez arterial (8), aumento da espessura da parede da artéria, entre outras modificações. Estudos casocontrole conduzidos em várias populações específicas têm demonstrado a influência deletéria do envelhecimento e outros fatores de risco como a hipertensão arterial e o diabetes mellitus, sobre as propriedades funcionais e estruturais das grandes artérias $(8,9)$.

Tanto o diabetes tipo 1 quanto o tipo 2 são associados com excesso de doença cardiovascular (DCV), onde as principais causas de mortalidade são conseqüência de lesões ateroscleróticas de grandes artérias como o infarto do miocárdio e o acidente vascular cerebral. Diversos estudos têm demonstrado alterações funcionais e estruturais de grandes artérias em diabéticos, principalmente aumento da rigidez arterial em diabéticos tanto do tipo 1 (10-13) quanto do tipo 2 (14-19).

Neste artigo, iremos descrever as alterações das propriedades funcionais e estruturais das grandes artérias que ocorrem freqüientemente no diabetes mellitus, tanto

MEDIDA DA VELOCIDADE DE ONDA DE PULSO
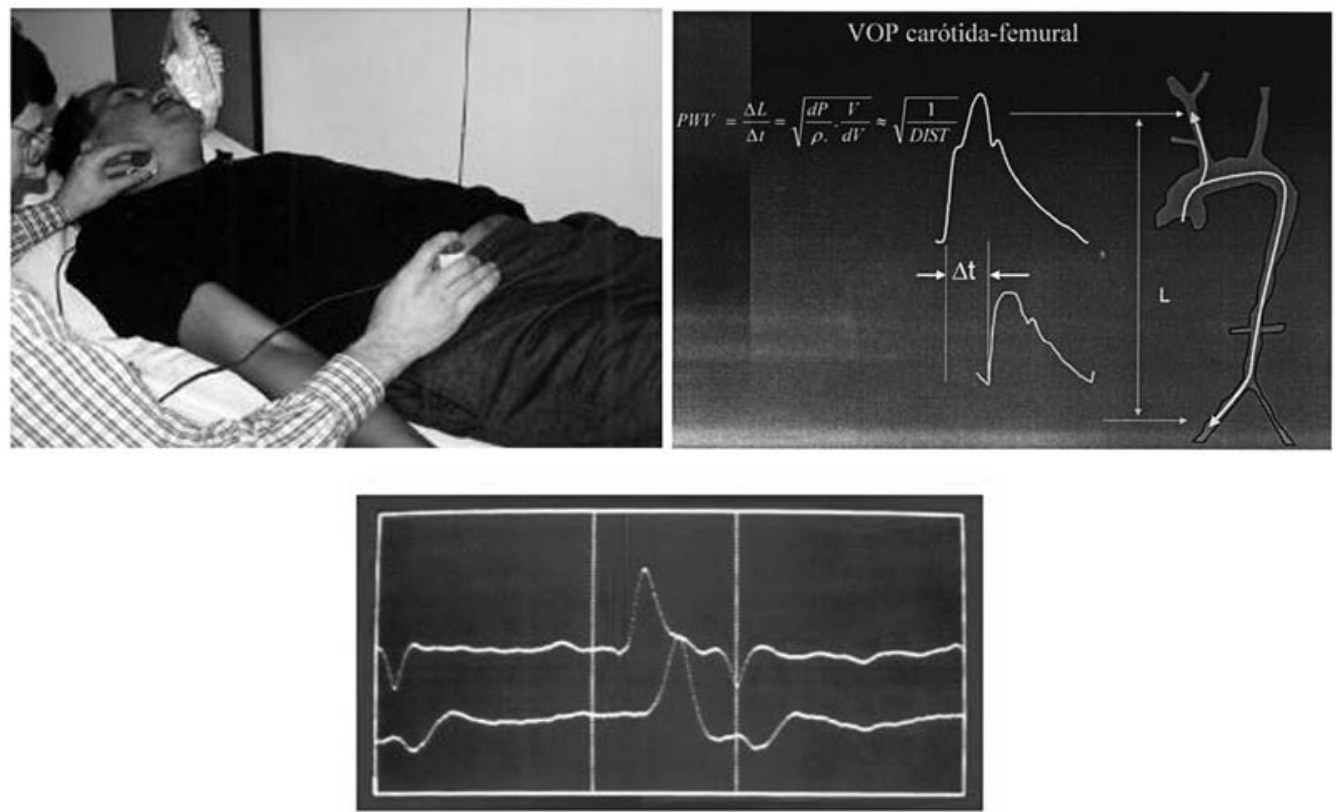

Figura 1. Esquema reproduzindo a técnica da medida da velocidade de onda de pulso aórtica por método não-invasivo. Na foto à esquerda, o posicionamento dos sensores mecanográficos nas artérias carótida e femural. Na figura à direita, a fórmula baseada na equação de Moertweg, na qual a velocidade de onda de pulso (VOP) está inversamente relacionada à distensibilidade, e o esquema utilizado para a medida. Na foto abaixo, a imagem visível na tela com as curvas de onda de pulso captadas na carótida e na femural. 
no tipo 1 quanto no tipo 2 , com destaque para as alterações da rigidez arterial. Além disso, abordaremos os possíveis mecanismos dessas alterações, as implicações das mesmas sobre as complicações cardiovasculares e renais do diabetes, e como o tratamento pode modificá-las.

\section{ALTERAÇÕES DAS PROPRIEDADES VASCULARES NO DIABETES TIPO 1}

O risco de DCV em pacientes com diabetes tipo 1 é particularmente elevado na presença de nefropatia, mas também é elevado nos pacientes sem envolvimento renal do diabetes. Tem sido evidenciado que pacientes com diabetes tipo 1 têm artérias mais rígidas do que indivíduos não-diabéticos da mesma faixa etária, e que este processo de enrijecimento arterial se inicia antes que qualquer sinal das complicações microvasculares ou macrovasculares possa ser detectado $(10,11)$. Essas alterações, inclusive, parecem ocorrer precocemente, pois crianças pré-púberes já apresentam aumento de rigidez arterial, demonstrado por tonometria de artéria braquial, quando comparadas com crianças saudáveis da mesma idade (20).

Este aumento da rigidez arterial encontrado em pacientes com diabetes tipo l parece ser correlacionado com a duração da doença, independentemente da idade (13), e tem repercussões sobre o comportamento da pressão arterial destes pacientes. Em um estudo populacional (12) realizado na Finlândia (FinnDiane) envolvendo cerca de 3.000 pacientes com diabetes tipo $1 \mathrm{e}$ 5.500 controles não-diabéticos, demonstrou-se que os indivíduos diabéticos tinham maior pressão arterial sistólica em todos os grupos etários, e a pressão diastólica foi maior em diabéticos abaixo de 40 anos e menor naqueles acima de 45 , caracterizando uma pressão de pulso mais elevada nos indivíduos diabéticos. Além disso, o estudo demonstrou que esse aumento prematuro da pressão de pulso é fortemente relacionado ao tempo de exposição à hiperglicemia e ao desenvolvimento de nefropatia diabética, embora essa alteração da pressão de pulso tenha sido encontrada também nos pacientes sem microalbuminúria. Em outro estudo europeu (21), envolvendo 3.250 pacientes com diabetes tipo 1 , demonstrou-se que a pressão de pulso foi associada com a idade, mas também com a presença de complicações microvasculares do diabetes, tais como microalbuminúria e retinopatia. Os importantes resultados destes dois estudos suportam o conceito de um envelhecimento vascular precoce em portadores de diabetes tipo 1, visto que a pressão de pulso é um marcador de aumento da rigidez arterial.
A maioria dos estudos (11,22-26) tem demonstrado que os pacientes com diabetes tipo 1 apresentam artérias mais rígidas que indivíduos normais, mas vários importantes fatores, como idade do paciente, duração da doença, artéria estudada, sexo, o grau de controle com o tratamento, e as complicações da doença, podem influenciar os efeitos do diabetes tipo 1 sobre as grandes artérias. Assim, os resultados de alguns estudos são conflitantes. Analisando a distensibilidade aórtica em jovens com diabetes tipo $1 \mathrm{e}$ que apresentavam menos de 1 ano de diagnóstico, Lehmann e cols. (24) mostraram aortas mais distensíveis (78\%) nestes indivíduos. Em contrapartida, Kool e cols. (25) mostraram, em adultos diabéticos tipo 1 não complicados, uma redução de distensibilidade na artéria femural, mas não nas artérias carótida ou braquial. Outro estudo (26) relatou aumento da rigidez da carótida e da aorta em mulheres mas não em homens com diabetes tipo 1 , e nessas mulheres houve correlação entre rigidez aórtica, duração do diabetes e disfunção autonômica. Em um estudo italiano (11), os autores mostraram em pacientes com diabetes tipo 1 um enrijecimento e um espessamento da parede arterial difuso, mesmo em pacientes sem qualquer complicação clínica vascular, demonstrando que a hipertrofia e a rigidez de grandes artérias são marcadores precoces de dano vascular nesta patologia (figura 2). Quando todos os dados publicados são considerados em conjunto, com exceção de um estudo, todos os demais mostram diminuição da distensibilidade arterial no diabetes tipo 1 . Essas anormalidades arteriais, relatadas em crianças, adolescentes e pacientes jovens, parecem ser mais pronunciadas na aorta do que nas extremidades inferiores, com uma correlação significante com a exposição do vaso à hiperglicemia.

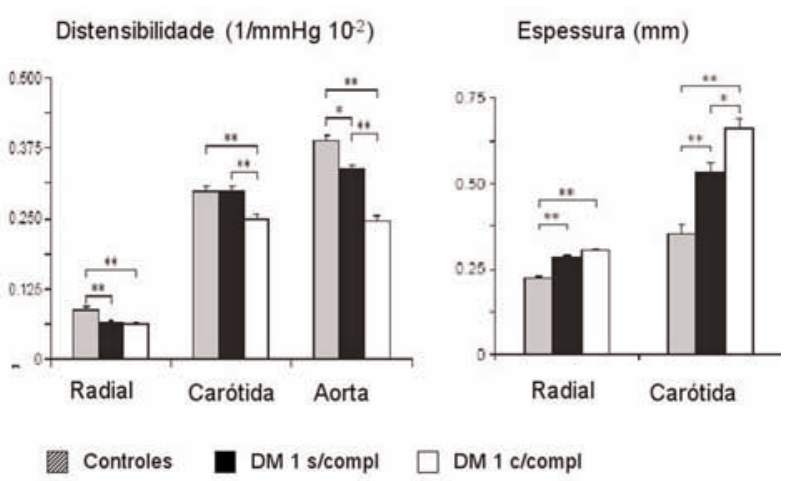

Figura 2. Distensibilidade arterial e espessura da parede arterial de indivíduos controles, diabéticos tipo 1 sem complicações (DM $1 \mathrm{~s} / \mathrm{compl}$ ) microvasculares e diabéticos tipo $1 \mathrm{com}$ complicações microvasculares (DM $1 \mathrm{c} / \mathrm{compl}$ ) (Giannatasio e cols., ref. 25). 
Com relação às alterações estruturais, tem sido demonstrado que pacientes diabéticos tipo 1 , desde a infầncia (27) até aqueles pacientes com complicações associadas (28), apresentam maior espessura intima medial (EIM) de artéria carótida do que os indivíduos sem a doença da mesma faixa etária. Em um desses estudos houve uma correlação importante entre EIM de carótida e disfunção endotelial, sugerindo a participação das alterações do endotélio no desenvolvimento de aterosclerose destes pacientes.

\section{ALTERAÇÕES DAS PROPRIEDADES VASCULARES NO DIABETES TIPO 2}

Assim como em pacientes com diabetes tipo 1 , vários estudos mostraram alterações da estrutura e função de grandes artérias em indivíduos com diabetes tipo 2, principalmente aumento da rigidez aórtica e da EIM da carótida $(15,19,22,29-32)$, sugerindo que a rigidez arterial pode contribuir para aterosclerose acelerada no diabetes tipo 2. Em um desses estudos, Lehmann e cols. (24) analisaram a complacência aórtica em pacientes diabéticos tipo 2 utilizando medidas de VOP, e observaram que os diabéticos tipo 2 tinham aortas mais rígidas do que os controles não-diabéticos de mesmo sexo e idade. No Strong Heart Study (33), a rigidez arterial foi medida por ultra-som em indígenas americanos, sendo 1.810 diabéticos e 944 normoglicêmicos. Os pacientes diabéticos tinham uma rigidez arterial significativamente aumentada em relação aos não-diabéticos e, além disso, a condição "diabetes" foi independentemente associada com a rigidez mes- mo após ajustes para idade, sexo, altura, índice de massa corpórea, pressão arterial sistólica e uso de medicação anti-hipertensiva.

Outro importante estudo (17), envolvendo 619 holandeses (Hoorn Study), avaliou a distensibilidade central e periférica em indivíduos com metabolismo de glicose normal, em indivíduos com intolerância à glicose e em pacientes com diabetes tipo 2. Tanto a rigidez de artérias centrais quanto de artérias periféricas estavam mais elevadas nos pacientes com intolerância à glicose e nos diabéticos, em comparação com os controles normais (figura 3 ).

$\mathrm{Na}$ maioria dos estudos de rigidez arterial em diabetes, os melhores determinantes da rigidez arterial foram a idade e os níveis de insulina plasmática, mas também observou-se correlação com a área sob a curva glicêmica. A presença de diabetes em pacientes com outros fatores de risco cardiovascular, como hipertensão arterial, tem efeito deletério sobre a já comprometida função vascular. Neste sentido, Amar e cols. (19) demonstraram em hipertensos não tratados que os portadores de diabetes e de intolerância à glicose apresentavam valores mais elevados de VOP do que naqueles com glicemia normal, havendo uma correlação positiva entre VOP e glicemia de jejum. Em estudo de nosso grupo envolvendo pacientes com hipertensão arterial leve/moderada, dentre diferentes determinantes, incluindo sensibilidade ao sal, os níveis de glicemia foram independentemente preditores da VOP aórtica (34).

As alterações funcionais das grandes artérias em pacientes diabéticos do tipo 2 parecem sofrer influência do sexo. No estudo de Westerbacka e cols.

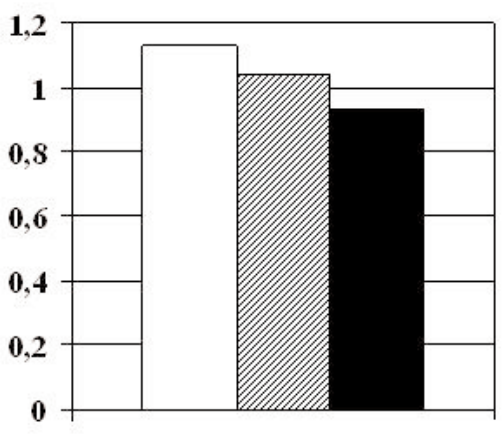

$\square$ Normal ITG $\square$ DM 2

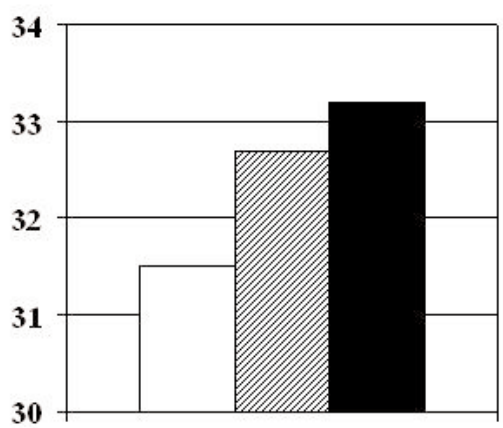

$\square$ Normal

Figura 3. Valores de complacência arterial sistêmica total e do índice de incremento aórtico, ambos índices de rigidez arterial em indivíduos normais (243), com intolerância à glicose (ITG-129) e diabéticos tipo 2 (DM2-156) do estudo Hoorn (Schram e cols., ref. 13). 
(35), a rigidez arterial, avaliada pelo índice de incremento da pressão central de aorta obtido por tonometria de aplanação de carótida, foi mais elevada em mulheres do que em homens. Em outro estudo (36), envolvendo 186 indivíduos diabéticos e nãodiabéticos, as mulheres diabéticas apresentaram VOP mais elevada que as não-diabéticas, enquanto entre os homens a VOP não diferiu entre os diabéticos e nãodiabéticos após ajuste para pressão arterial. Além disso, o enrijecimento da aorta relacionado à idade foi maior apenas em mulheres diabéticas. Assim, em homens, a presença de diabetes tipo 2 não é necessariamente responsável por uma maior rigidez arterial além do efeito da hipertensão arterial concomitante, enquanto em mulheres o diabetes tipo 2 é associado a um aumento acelerado da rigidez arterial relacionado à idade além do esperado para um determinado nível de pressão arterial.

Independentemente dessas diferenças relacionadas ao sexo, as alterações da rigidez arterial presentes em diabéticos tipo 2 ocorrem precocemente, mesmo na ausência de outros fatores de risco ou de manifestações clínicas de doença cardiovascular. Em outro estudo de nosso grupo (18), demonstramos uma elevação da VOP da aorta, artéria predominantemente elástica, e também da VOP da artéria braquial, predominantemente muscular, em pacientes diabéticos tipo 2 sem nenhuma manifestação clínica de doença cardiovascular ou fator de risco associado (figura 4). Nesse mesmo estudo, a VOP aórtica foi significativamente relacionada aos níveis de glicemia e à hemoglobina glicosilada, salientando a participação do estado hiperglicêmico nas alterações da função vascular desses pacientes.

Assim como já demonstrado em pacientes hipertensos (5) ou com insuficiência renal crônica dialítica, a rigidez arterial avaliada pela VOP aórtica foi um marcador de eventos e mortalidade cardiovascular

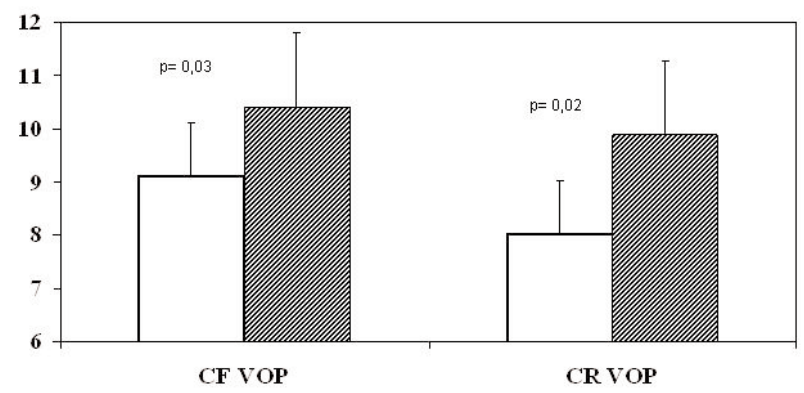

Figura 4. Valores de velocidade de onda de pulso (VOP) em $\mathrm{m} / \mathrm{s}$ carótida-femural (CF) e carótida-radial (CR), ajustados pela idade em pacientes com diabetes (barras cruzadas) e em indivíduos controles (barras brancas) (Bortolotto e cols., ref. 14). de indivíduos com diabetes tipo 2 (37). Neste estudo, realizado em 397 pacientes diabéticos de diferentes etnias, a VOP aórtica também foi maior nos indivíduos diabéticos comparados aos normoglicêmicos, e a mortalidade em 10 anos foi mais elevada entre os diabéticos. Além disso, a VOP aórtica, ao lado de idade, tabagismo e valor da curva glicêmica, foi um preditor independente de mortalidade total, deslocando a pressão arterial sistólica quando ambos foram inseridos no mesmo modelo. Os pacientes diabéticos que morreram eram significativamente mais velhos, fumavam mais, tinham uma VOP $2,6 \mathrm{~m} / \mathrm{s}$ mais elevada e a pressão arterial média era $10 \mathrm{mmHg}$ mais alta que a daqueles que não morreram (tabela $\mathrm{l}$ ).

Com relação às alterações estruturais, tem sido consistentemente demonstrado que a EIM de carótida é maior nos indivíduos com diabetes tipo 2 do que nos não-diabéticos (16,38-40). Em um desses estudos (40), Insulin Resistance Atherosclerosis Study, que envolveu 1.625 pacientes em vários graus de estado hiperglicêmico, isto é, normoglicêmico, intolerância à glicose, diabetes recém-diagnosticado e estabelecido, a EIM de carótida foi significativamente maior nos estados diabéticos (figura 5). Além disso, o diabetes mellitus é fator importante de progressão da EIM de carótida, como foi demonstrado em estudo (41) realizado em 1.192 indivíduos, onde o aumento da espessura intima medial de carótida após 5 anos de seguimento foi 2 vezes maior na população diabética do que nos normoglicêmicos, após correção para outros fatores de risco.

Tabela 1. Variáveis clínicas e hemodinâmicas de 397 pacientes com diabetes mellitus tipo 2 de acordo com a mortalidade no seguimento de 10 anos em média.

$\begin{array}{ccc}\text { Vivos } & \text { Mortos } & p \\ (n=218) & (n=179)\end{array}$

\begin{tabular}{lccc}
$\begin{array}{l}\text { Idade, } \\
\text { anos, média }\end{array}$ & 57,5 & 63,8 & $<0,05$ \\
$\begin{array}{l}\text { Sexo, } \\
\text { M/F, (\%) }\end{array}$ & $55 / 45$ & $66 / 34$ & $\mathrm{~ns}$ \\
$\begin{array}{l}\text { Tabagismo, } \\
\text { S/N, (\%) }\end{array}$ & $47 / 53$ & $63 / 37$ & $<0,05$ \\
$\begin{array}{l}\text { Duração do diabetes, } \\
\text { anos, média }\end{array}$ & 5,7 & 7,8 & $<0,05$ \\
$\begin{array}{l}\text { PA sistólica, } \\
\text { mmHg, média }\end{array}$ & 140 & 150 & $<0,05$ \\
$\begin{array}{l}\text { VOP aórtica, } \\
\text { m/s, média }\end{array}$ & 10,4 & 13,0 & $<0,05$ \\
\hline
\end{tabular}




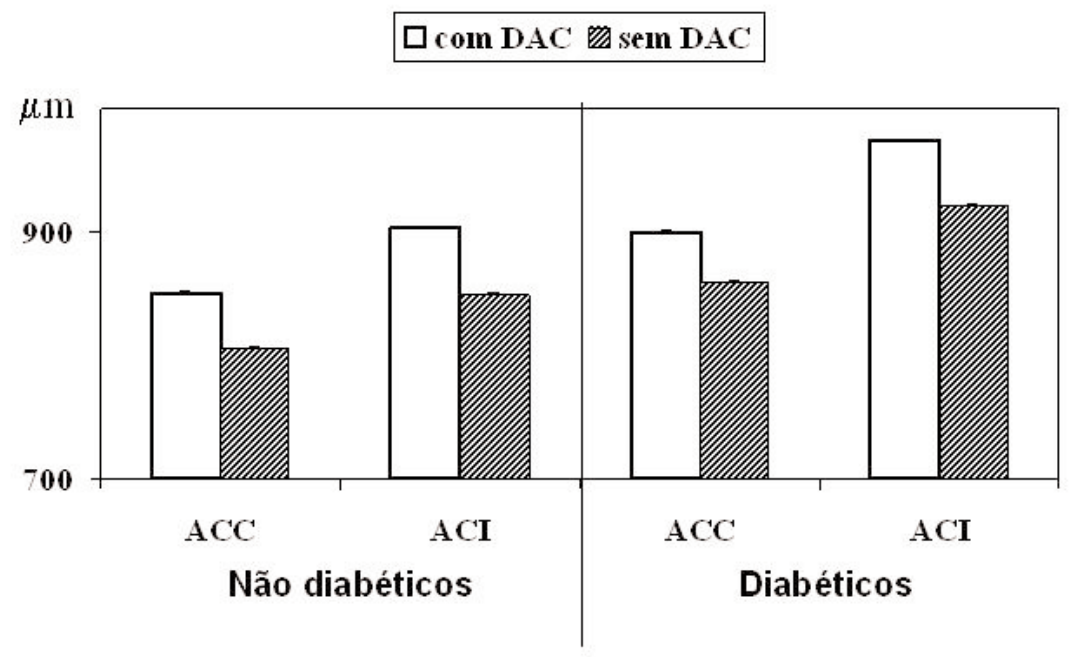

$\mathrm{ACC}$ e $\mathrm{ACl}$ - Diabéticos vs. Nấo-diabéticos $-p<0,05$ ACC - DAC vs. Sem DAC $-p<0,05$

\begin{abstract}
Figura 5. Valores de espessura intima-medial da artéria carótida comum (ACC) e da artéria carótida interna $(\mathrm{ACl})$, de pacientes diabéticos tipo II e indivíduos controles com e sem doença coronariana (DAC) - Estudo IRAS (Insulin Resistance Atherosclerosis Study) (Haffner e cols., ref. 38).
\end{abstract}

A EIM de carótida também tem valor prognóstico cardiovascular em pacientes diabéticos tipo 2 . A EIM aumentada está associada com doença arterial coronária e é preditora de eventos futuros de infarto do miocárdio silencioso e doença coronariana em diabéticos tipo $2(42)$.

Ambas as alterações funcionais e estruturais parecem estar inter-relacionadas em pacientes diabéticos, como foi observado em um estudo (14) realizado em 225 indivíduos com diabetes tipo 2 , onde a rigidez arterial avaliada pela análise da onda de pulso por tonometria de aplanação foi significativamente e independentemente relacionada à EIM da artéria carótida.

\section{MECANISMOS DE AUMENTO DA RIGIDEZ ARTERIAL E DAS ALTERAÇÕES VASCULARES ESTRUTURAIS EM PACIENTES DIABÉTICOS}

As alterações da função vascular presentes tanto no diabetes tipo 2 quanto no diabetes tipo 1 têm vários mecanismos fisiopatológicos possíveis. Um dos mecanismos seria relacionado ao aumento da resistência à insulina, e conseqüente hiperinsulinemia. A insulina reduz significativamente a rigidez de grandes artérias quando infundida agudamente (30 a $60 \mathrm{~min}$ ), provavelmente por um mecanismo endotélio-dependente, como demonstrado em indivíduos jovens saudáveis (35), sugerindo que hiperinsulinemia pode promover também um aumento da rigidez arterial no período pós-prandial por disfunção endotelial. A associação de resistência à insulina e aumento da rigidez arterial tem sido demonstrada em indivíduos com diabetes mellitus tipo 2 , onde a captação de glicose mediada por insulina foi positivamente associada com o coeficiente de complacência da artéria carótida (43).

A hiperglicemia também pode diretamente afetar a estrutura e, conseqüentemente, a função de grandes artérias. Tem sido demonstrado que a hiperglicemia causa importantes alterações quantitativas e qualitativas na elastina e no colágeno de paredes de artérias centrais (17). Outro possível mecanismo que pode permitir elevação da rigidez arterial nos pacientes diabéticos é a glicação de proteínas e o aumento de produtos finais de glicação avançada. Esses produtos podem formar ligações de moléculas de colágeno na parede arterial, desta forma diminuindo a distensibilidade (17). Condições hiperglicêmicas podem promover acúmulo de colágeno devido a uma glicação não-enzimática e conseqüentemente aumentar a rigidez de artérias centrais e periféricas (44). Por fim, resultados de estudo desenvolvido em nosso laboratório sugerem que alterações do sistema nervoso autônomo podem induzir as al- 
terações da rigidez arterial presentes nos pacientes diabéticos. Neste estudo, demonstramos (18) que pacientes diabéticos sem complicações cardiovasculares ou renais apresentavam elevações da VOP aórtica e braquial correlacionadas com alterações do reflexo autonômico simpático, principalmente aquelas dependentes dos barorreceptores, sugerindo a participação do sistema nervoso autônomo na mediação das alterações da rigidez arterial presentes em pacientes com diabetes.

O aumento da EIM da carótida pode ser desencadeado pela hiperglicemia, como descrito anteriormente, com conseqüente aumento da quantidade de colágeno e elastina da parede arterial. Mas esse mecanismo não deve ser o único, pois há evidências de participação da inflamação e da genética nas alterações estruturais de grandes vasos presentes no diabetes. Com relação à inflamação, achados de estudo (45) realizado em diabéticos tipo 2 mostraram que a extensão da espessura intima medial de carótida foi relacionada à proteína $\mathrm{C}$ reativa e ao fibrinogênio. A participação genética no desenvolvimento da lesão estrutural vascular de pacientes diabéticos tipo 2 foi sugerida por Yazdanpanah e cols. (46), que recentemente demonstraram maior EIM de carótida em pacientes com diabetes tipo 2 que apresentavam polimorfismo genético da a-aducina 1. Esse polimorfismo se caracteriza pelo aumento da atividade da bomba sódio-potássio, aumentando a reabsorção tubular renal de sódio e conseqüentemente elevando o risco de hipertensão sal-sensível e de doença vascular associada.

\section{RELAÇĀO ENTRE RIGIDEZ ARTERIAL E COMPLICAÇŌES CARDIOVASCULARES E RENAIS DOS PACIENTES COM DIABETES MELLITUS}

Lesão vascular generalizada deve ser um mecanismo patogênico comum ligando as complicações renais e, o desenvolvimento de aterosclerose no paciente diabético. Além disso, a presença de alterações funcionais e estruturais de grandes artérias pode piorar o prognóstico de pacientes com diabetes e outras complicações, principalmente as renais, manifestas por microalbuminúria ou insuficiência renal. Pacientes com doença renal terminal e diabetes têm uma VOP aórtica mais elevada do que aqueles indivíduos sem diabetes, e essa condição contribui para a maior mortalidade e morbidade cardiovascular desses pacientes (47). Uma relação positiva entre pressão de pulso, um marcador clínico de elevada rigidez arterial, e proteinúria tem sido observada (48), e, em um recente estudo em pacientes japoneses com diabetes tipo 2 , a microalbuminúria foi independentemente relacionada à EIM da artéria carótida (49). Em outro estudo recentemente publicado, Smith e cols. (50) demonstraram que pacientes com diabetes tipo 2 que apresentam uma razão albumina/creatinina elevada têm uma maior rigidez aórtica que os indivíduos com razão menor, e que os valores de pressão arterial elevados desses pacientes com excreção de albumina maior são os mediadores dessa correlação. Além disso, nesse mesmo estudo, em pacientes com diabetes tipo 2 sem lesão renal manifesta, o ritmo de filtração glomerular (RFG) foi negativamente associado com a VOP aórtica. Esses dados reforçam os obtidos por Taniwaki e cols. (51), que demonstraram que o RFG foi negativamente e independentemente relacionado à rigidez de artéria carótida. Os dados desses dois estudos sugerem que em diabetes tipo 2, o nível de função renal, bem antes da insuficiência renal, é um importante fator contribuinte para a rigidez arterial. Assim, alterações funcionais renais precoces per se parecem interagir não somente com a função de pequenas mas também de grandes artérias, contribuindo para o desenvolvimento e progressão do envelhecimento vascular e do processo aterosclerótico dos pacientes diabéticos.

Com relação às complicações cardiovasculares, alguns autores (52) têm demonstrado que a maior rigidez de artérias de membros inferiores está associada com fluxo arterial diastólico de artéria poplítea diminuído, à isquemia de pé induzida por exercício e a sintomas isquêmicos de doença arterial periférica em pacientes com diabetes mellitus tipo 2. Também se tem demonstrado que doença arterial coronária com manifestações isquêmicas está relacionada com aumento da VOP aórtica e não de outros territórios arteriais em pacientes diabéticos, indicando a participação da rigidez de artérias centrais no mecanismo de isquemia miocárdica de pacientes com diabetes tipo 2 (53).

\section{EFEITOS DO TRATAMENTO SOBRE A RIGIDEZ ARTERIAL}

O tratamento do diabetes per se, ou o tratamento de co-morbidades associadas ao diabetes como hipertensão e dislipidemia, pode promover melhora das alterações estruturais e funcionais das grandes artérias em pacientes diabéticos. Embora não existam muitos estudos abordando esses tópicos, algumas publicações têm mostrado resultados auspiciosos que incentivam a realização de estudos com maior número de participantes. $\mathrm{O}$ tratamento de pacientes diabéticos 
com glitazonas ou metformina melhora a função vascular de pacientes diabéticos via aumento de adiponectina, permitindo um entendimento melhor dos mecanismos dos efeitos do tratamento sobre as alterações vasculares. Tem-se demonstrado efeitos benéficos do uso de estatinas sobre a rigidez arterial regional de membros inferiores de pacientes com diabetes tipo 2 , independentemente da redução dos níveis de colesterol (54). Em estudo (55) recentemente publicado, os autores demonstraram em 42 pacientes normotensos com diabetes tipo 1 ou tipo 2 , que o uso por 6 meses de inibidor da enzima conversora reduziu significativamente a VOP aórtica independentemente do efeito sobre a pressão arterial, sobretudo nos pacientes com diabetes tipo 1 . Confirmando esses dados, Tropeano e cols. (56) demonstraram em 57 pacientes com hipertensão e diabetes tipo 2 que o uso de inibidor de enzima conversora por 7 meses aumentou significativamente a distensibilidade de carótida independentemente do efeito sobre a artéria braquial.

\section{CONCLUSÃO}

Pacientes portadores de diabetes mellitus tipo 1 e tipo 2 apresentam alterações estruturais e funcionais de grandes vasos que muito provavelmente têm um papel fundamental no desenvolvimento de aterosclerose desses pacientes e suas respectivas complicações cardiovasculares. A possibilidade de detectar precocemente essas alterações através de métodos não-invasivos permite identificar os pacientes de maior risco que se beneficiarão de um tratamento mais agressivo. Além disso, abre-se uma perspectiva nova de abordagem terapêutica dos pacientes diabéticos, com a adoção de medidas nãofarmacológicas ou farmacológicas que interfiram nessas modificações vasculares e conseqüentemente ajudem a diminuir a mortalidade cardiovascular desses pacientes.

\section{REFERÊNCIAS}

1. Stefanadis C, Stratos C, Boudoulas H, Kourouklis C, Toutouzas P. Distensibility of the ascending aorta: comparison of invasive and non-invasive techniques in healthy men and in men with coronary artery disease. Eur Heart $\mathbf{J}$ 1990;11(11):990-6.

2. Hirai T, Sasayama S, Kawasaki T, Yagi S. Stiffness of systemic arteries in patients with myocardial infarction. A noninvasive method to predict severity of coronary atherosclerosis. Circulation 1989;80(1):78-86.

3. Bogren HG, Mohiaddin RH, Klipstein RK, Firmin DN, Underwood RS, Rees SR, et al. The function of the aorta in ischemic heart disease: a magnetic resonance and angiographic study of aortic compliance and blood flow patterns. Am Heart J 1989;118(2):234-47.
4. Shimojo M, Tsuda N, Iwasaka T, Inada M. Age-related changes in aortic elasticity determined by gated radionuclide angiography in patients with systemic hypertension or healed myocardial infarcts and in normal subjects. Am J Cardiol 1991;68(9):950-3.

5. Laurent S, Boutouyrie P, Asmar R, Gautier I, Laloux B, Guize $\mathrm{L}$, et al. Aortic stiffness is an independent predictor of allcause and cardiovascular mortality in hypertensive patients. Hypertension 2001;37(5):1236-41.

6. Bortolotto LA, Blacher J, Kondo T, Takazawa K, Safar ME. Assessment of vascular aging and atherosclerosis in hypertensive subjects: second derivative of photoplethysmogram versus pulse wave velocity. Am J Hypertens 2000;13(2):165-71.

7. Bortolotto LA, Safar ME, Billaud E, Lacroix C, Asmar R, London GM, et al. Plasma homocysteine, aortic stiffness, and renal function in hypertensive patients. Hypertension 1999;34(4 Pt 2):837-42.

8. Safar ME, Levy BI, Struijker-Boudier H. Current perspectives on arterial stiffness and pulse pressure in hypertension and cardiovascular diseases. Circulation 2003;107(22):2864-9.

9. Benetos A, Waeber B, Izzo J, Mitchell G, Resnick L, Asmar R, et al. Influence of age, risk factors, and cardiovascular and renal disease on arterial stiffness: clinical applications. Am J Hypertens 2002;15(12):1101-8.

10. Berry KL, Skyrme-Jones RA, Cameron JD, O'Brien RC, Meredith IT. Systemic arterial compliance is reduced in young patients with IDDM. Am J Physiol 1999;276/6 Pt 2): $\mathrm{H} 1839-45$

11. Giannattasio C, Failla M, Piperno A, Grappiolo A, Gamba P, Paleari $F$, et al. Early impairment of large artery structure and function in type I diabetes mellitus. Diabetologia 1999;42(8):987-94.

12. Ronnback M, Fagerudd J, Forsblom C, Pettersson-Fernholm K, Reunanen A, Groop PH. Altered age-related blood pressure pattern in type 1 diabetes. Circulation 2004;110(9):1076-82.

13. Wilkinson IB, MacCallum H, Rooijmans DF, Murray GD, Cockcroft JR, McKnight JA, et al. Increased augmentation index and systolic stress in type 1 diabetes mellitus. OJM 2000;93(7):441-8.

14. Westerbacka J, Leinonen E, Salonen JT, Salonen R, Hiukka A, Yki-Jarvinen $\mathrm{H}$, et al. Increased augmentation of central blood pressure is associated with increases in carotid intima-media thickness in type 2 diabetic patients. Diabetologia 2005;48(8):1654-62.

15. Wahlqvist ML, Lo CS, Myers KA, Simpson RW, Simpson JM. Putative determinants of arterial wall compliance in NIDDM. Diabetes Care 1988;11(10):787-90.

16. Wagenknecht LE, D'Agostino Jr. RB, Haffner SM, Savage PJ, Rewers M. Impaired glucose tolerance, type 2 diabetes, and carotid wall thickness: the Insulin Resistance Atherosclerosis Study. Diabetes Care 1998;21(11):1812-8.

17. Schram MT, Henry RM, van Dijk RA, Kostense PJ, Dekker JM, Nijpels G, et al. Increased central artery stiffness in impaired glucose metabolism and type 2 diabetes: the Hoorn Study. Hypertension 2004;43(2):176-81.

18. Bortolotto LG, Lopes H, Guerra-Riccio G, Krieger E, Malachias MVB. Increased arterial stiffness and impaired baroreflex sensitivity are correlated in normotensive individuals with type 2 diabetes. J Hypertens 2004;22(suppl 1):63S.

19. Amar J, Chamontin B, Pelissier M, Garelli I, Salvador M. Influence of glucose metabolism on nycthemeral blood pressure variability in hypertensives with an elevated waist-hip ratio. A link with arterial distensibility. Am J Hypertens 1995;8(4 Pt 1):426-8.

20. Haller MJ, Samyn M, Nichols WW, Brusko T, Wasserfall C, Schwartz RF, et al. Radial artery tonometry demonstrates arterial stiffness in children with type 1 diabetes. Diabetes Care 2004;27(12):2911-7.

21. Schram MT, Chaturvedi N, Fuller JH, Stehouwer CD. Pulse pressure is associated with age and cardiovascular disease in type 1 diabetes: the Eurodiab Prospective Complications Study. J Hypertens 2003;21(11):2035-44. 
22. Woolam GL, Schnur PL, Vallbona C, Hoff HE. The pulse wave velocity as an early indicator of atherosclerosis in diabetic subjects. Circulation 1962;25:533-9.

23. Okada M, Matsuto $T$, Satoh S, Igarashi S, Baba M, Sugita O. Role of pulse wave velocity for assessing autonomic nervous system activities in reference to heart rate variability. Med Inform (Lond) 1996;21(1):81-90.

24. Lehmann ED, Watts GF, Fatemi-Langroudi B, Gosling RG. Aortic compliance in young patients with heterozygous familial hypercholesterolaemia. Clin Sci (Lond) 1992;83(6):717-21.

25. Kool M, Lustermans F, Kragten H, Struijker Boudier H, Hoeks A, Reneman $\mathrm{R}$, et al. Does lowering of cholesterol levels influence functional properties of large arteries? Eur J Clin Pharmacol 1995;48(3-4):217-23.

26. Ryden Ahlgren A, Lanne T, Wollmer $P$, Sonesson B, Hansen F, Sundkvist G. Increased arterial stiffness in women, but not in men, with IDDM. Diabetologia 1995;38(9):1082-9.

27. Jarvisalo MJ, Putto-Laurila A, Jartti L, Lehtimaki T, Solakivi T, Ronnemaa $\mathrm{T}$, et al. Carotid artery intima-media thickness in children with type 1 diabetes. Diabetes 2002;51(2):493-8.

28. Krantz JS, Mack WJ, Hodis HN, Liu CR, Liu CH, Kaufman FR. Early onset of subclinical atherosclerosis in young persons with type 1 diabetes. J Pediatr 2004:145(4):452-7.

29. Megnien JL, Simon A, Valensi P, Flaud P, Merli I, Levenson J. Comparative effects of diabetes mellitus and hypertension on physical properties of human large arteries. J Am Coll Cardiol 1992;20(7):1562-8.

30. Tanokuchi S, Okada S, Ota Z. Factors related to aortic pulsewave velocity in patients with non-insulin-dependent diabetes mellitus. J Int Med Res 1995;23(6):423-30.

31. Hopkins KD, Lehmann ED, Jones RL, Turay RC, Gosling RG. A family history of NIDDM is associated with decreased aortic distensibility in normal healthy young adult subjects. Diabetes Care 1996;19(5):501-3.

32. Emoto M, Nishizawa Y, Kawagishi T, Maekawa K, Hiura Y, Kanda $\mathrm{H}$, et al. Stiffness indexes beta of the common carotid and femoral arteries are associated with insulin resistance in NIDDM. Diabetes Care 1998;21(7):1178-82.

33. Devereux RB, Roman MJ, Paranicas M, O'Grady MJ, Lee ET, Welty TK, et al. Impact of diabetes on cardiac structure and function: the strong heart study. Circulation 2000;101(19):2271-6.

34. Dórea EL, Bortolotto LA, Bernik M, Krieger JE. Efeitos da sensibilidade ao sal e obesidade na distensibilidade vascular de pacientes hipertensos não-diabéticos. Hipertensão 2005;8(Suppl):8.

35. Westerbacka J, Vehkavaara S, Bergholm R, Wilkinson I, Cockcroft J, Yki-Jarvinen $\mathrm{H}$. Marked resistance of the ability of insulin to decrease arterial stiffness characterizes human obesity. Diabetes 1999;48(4):821-7.

36. De Angelis L, Millasseau SC, Smith A, Viberti G, Jones RH, Ritter JM, et al. Sex differences in age-related stiffening of the aorta in subjects with type 2 diabetes. Hypertension 2004;44(1):67-71.

37. Cruickshank K, Riste L, Anderson SG, Wright JS, Dunn G, Gosling RG. Aortic pulse-wave velocity and its relationship to mortality in diabetes and glucose intolerance: an integrated index of vascular function? Circulation 2002;106(16):2085-90.

38. Mohan V, Ravikumar R, Shanthi Rani S, Deepa R. Intimal medial thickness of the carotid artery in South Indian diabetic and non-diabetic subjects: the Chennai Urban Population Study (CUPS). Diabetologia 2000;43(4):494-9.

39. Temelkova-Kurktschiev TS, Koehler C, Leonhardt W, Schaper F, Henkel E, Siegert G, et al. Increased intimal-medial thickness in newly detected type 2 diabetes: risk factors. Diabetes Care 1999;22(2):333-8.

40. Haffner SM, Agostino Jr. RD, Saad MF, O'Leary DH, Savage PJ, Rewers M, et al. Carotid artery atherosclerosis in type-2 diabetic and nondiabetic subjects with and without symptomatic coronary artery disease (The Insulin Resistance Atherosclerosis Study). Am J Cardiol 2000;85(12):1395-400.

41. Wagenknecht LE, Zaccaro D, Espeland MA, Karter AJ, O'Leary DH, Haffner SM. Diabetes and progression of carotid atherosclerosis: the insulin resistance atherosclerosis study. Arterioscler Thromb Vasc Biol 2003;23(6):1035-41.
42. Yokoyama H, Katakami N, Yamasaki Y. Recent advances of intervention to inhibit progression of carotid intima-media thickness in patients with type 2 diabetes mellitus. Stroke 2006;37(9):2420-7.

43. van Dijk RA, Bakker SJ, Scheffer PG, Heine RJ, Stehouwer $\mathrm{CD}$. Associations of metabolic variables with arterial stiffness in type 2 diabetes mellitus: focus on insulin sensitivity and postprandial triglyceridaemia. Eur J Clin Invest 2003;33(4):307-15.

44. Airaksinen KE, Salmela $\mathrm{PI}$, Linnaluoto $\mathrm{MK}$, Ikaheimo $\mathrm{MJ}$, Ahola K, Ryhanen LJ. Diminished arterial elasticity in diabetes: association with fluorescent advanced glycosylation end products in collagen. Cardiovasc Res 1993;27(6):942-5.

45. Corrado E, Rizzo M, Muratori I, Coppola G, Novo S. Association of elevated fibrinogen and $\mathrm{C}$-reactive protein levels with carotid lesions in patients with newly diagnosed hypertension or type II diabetes. Arch Med Res 2006;37(8):1004-9.

46. Yazdanpanah $M$, Sayed-Tabatabaei FA, Hofman A, Aulchenko YS, Oostra BA, Stricker BH, et al. The alphaadducin gene is associated with macrovascular complications and mortality in patients with type 2 diabetes. Diabetes 2006;55(10):2922-7.

47. Shoji T, Emoto M, Shinohara K, Kakiya R, Tsujimoto $Y$, Kishimoto $\mathrm{H}$, et al. Diabetes mellitus, aortic stiffness, and cardiovascular mortality in end-stage renal disease. J Am Soc Nephrol 2001;12(10):2117-24.

48. Pedrinelli R, Dell'Omo G, Penno G, Bandinelli S, Bertini A, Di Bello $V$, et al. Microalbuminuria and pulse pressure in hypertensive and atherosclerotic men. Hypertension 2000;35(1 Pt 1):48-54

48. Yokoyama H, Aoki T, Imahori M, Kuramitsu M. Subclinical atherosclerosis is increased in type 2 diabetic patients with microalbuminuria evaluated by intima-media thickness and pulse wave velocity. Kidney Int 2004;66(1):448-54.

50. Smith A, Karalliedde J, De Angelis L, Goldsmith D, Viberti G. Aortic pulse wave velocity and albuminuria in patients with type 2 diabetes. J Am Soc Nephrol 2005;16(4):1069-75.

51. Taniwaki H, Nishizawa $Y$, Kawagishi $T$, Ishimura E, Emoto M, Okamura $\mathrm{T}$, et al. Decrease in glomerular filtration rate in Japanese patients with type 2 diabetes is linked to atherosclerosis. Diabetes Care 1998;21(11):1848-55.

52. Kizu A, Koyama H, Tanaka S, Maeno T, Komatsu M, Fukumoto $S$, et al. Arterial wall stiffness is associated with peripheral circulation in patients with type 2 diabetes. Atherosclerosis 2003;170(1):87-91.

53. Hatsuda S, Shoji T, Shinohara K, Kimoto E, Mori K, Fukumoto $\mathrm{S}$, et al. Regional arterial stiffness associated with ischemic heart disease in type 2 diabetes mellitus. J Atheroscler Thromb 2006;13(2):114-21.

54. Shinohara K, Shoji T, Kimoto E, Yokoyama H, Fujiwara S, Hatsuda $S$, et al. Effect of atorvastatin on regional arterial stiffness in patients with type 2 diabetes mellitus. J Atheroscler Thromb 2005;12(4):205-10.

55. Manolis AJ, Iraklianou S, Pittaras A, Zaris M, Tsioufis K, Psaltiras G, et al. Arterial compliance changes in diabetic normotensive patients after angiotensin-converting enzyme inhibition therapy. Am J Hypertens 2005;18(1):18-22.

56. Tropeano Al, Boutouyrie $P$, Pannier $B$, Joannides $R$, Balkestein E, Katsahian S, et al. Brachial pressure-independent reduction in carotid stiffness after long-term angiotensin-converting enzyme inhibition in diabetic hypertensives. Hypertension 2006;48(1):80-6.

\section{Endereço para correspondência:}

Luiz Aparecido Bortolotto

Unidade de Hipertensão

Instituto do Coração (InCor)

Av. Dr. Enéas de Carvalho Aguiar 44

05403-000 São Paulo, SP

Fax: (1 1 ) 3069-5048

E-mail: hipluiz@incor.usp.br 